\title{
X-Ray Diffraction Studies on Alicyclic Saturated Discotic Liquid Crystals
}

\author{
H. W. Neuling and H. Stegemeyer \\ Department of Physical Chemistry, University Paderborn, D-4790 Paderborn, F. R. Germany \\ K. Praefcke* and B. Kohne \\ Department of Organic Chemistry, Technische Universität Berlin
}

Z. Naturforsch. 42 a, 631-635 (1987); received February 20, 1987

Dedicated to Professor Ernst Fischer on the occasion of his 65th birthday

The discotic liquid crystalline phase of a new class of disc-like molecules (hexa-O-alkanoylscyllo-inositoles) is studied by small angle X-ray scattering, DSC and optical observations. Comparison of these three methods allows the determination of the structural arrangement. $\mathrm{X}$-ray film exposures of oriented samples clearly demonstrate the hexagonal columnar ordered type of mesophases. A simple model calculation shows that the alkyl chains of neighbouring columns interpenetrate each other to a small extent.

\section{Introduction}

The different ways in which disc-like molecules can be arranged in the discotic mesomorphic state have recently been the subject of many investigations [1]. Some discotic phases have been defined earlier. For example, the columnar phases have been divided into four basic groups because of their different lattice structures; e.g. $D_{\text {hd }}, D_{\text {ho }}, D_{\text {rd }}$ and $\mathrm{D}_{\text {ob.d }}$ [2]. The series of hexa-O-alkanoyl-scyllo inositoles (HASI) is of interest because disc-like molecules with alicyclic saturated cores were recently found to form discotic phases $[3,10]$. The aim of this paper was to study various parameters of the mesogenic phases of those compounds. Especially the intercore distance $d$ of the columns, the temperature dependence of $d$ and the influence of the alkyl chain lengths on the mesomorphic behaviour have been measured.

\section{Experimental}

The compounds of the homologous series of HASI (alkyl chain length from $n=5$ to $n=11$ ) were synthesized and purified as described elsewhere $[3,9]$.

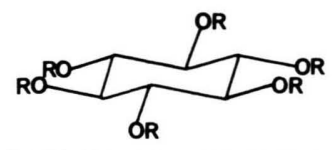

$$
\mathrm{R}=\mathrm{COC}_{n} \mathrm{H}_{2 n+1}
$$

* 42. Mitteilung über flüssigkistalline Verbindungen. Reprint requests to Prof. Dr. H. Stegemeyer, Department of Physical Chemistry, University Paderborn, P.O. Box 1621, D-4790 Paderborn, FRG.
The samples were filled in Lindemann capillaries (diameter $1 \mathrm{~mm}$ ) and then placed into a tight-fitting rectangular brass holder $(20 \times 5 \times 2 \mathrm{~mm})$. The orientation was performed using a magnetic field strength up to 2.4 Tesla in a Drusch electromagnet. The temperature and cooling rate $(0.2 \mathrm{~K} / \mathrm{min})$ was controlled by a modified Mettler FP 52 heating stage (all magnetic materials have been replaced by aluminium). The direction of the magnetic field was perpendicular to the long axis of the capillary. The $\mathrm{X}$-ray measurements were performed using small angle cameras of the type Kratky-Compact (A. Paar, Graz, Austria) and Kiessig (Seifert, Ahrensburg, FRG). We used film registration and detection by a curved linear position sensitive proportional counter PSPC (M. Braun, Munich) with an active length of $50 \mathrm{~mm}$ and a low flux of a mixture of $75 \%$ argon and $25 \%$ methane under a pressure of 11 bar. In order to calibrate the detector, the scattering curve of a cadmium-distearate multilayer (35 double layers) has been recorded at room temperature before each measurement. This calibration sample was prepared on a glass rod of $2 \mathrm{~mm}$ diameter using the Blodgett technique [4]. Its diffraction pattern consists of sharp reflections with a first order period of $d=(5.03 \pm 0.01) \mathrm{nm}$ [5]. Ni-filtered Cu-radiation was used. The temperature in the Kratkycompact camera was controlled by an electronic heating stage (A. Paar, Graz, Austria) with a relative accuracy of $\pm 0.02 \mathrm{~K}$. In the Kiessigcamera we used a thermostated water flow cell with

0932-0784 / 87 / 0600-0631 \$01.30/0. - Please order a reprint rather than making your own copy. 
$0.2 \mathrm{~K}$ accuracy. Both cameras were placed on the $\mathrm{X}$-ray tube window with point focus primary beam.

\section{Results and Discussion}

The phase transition temperatures of the HASI derivatives are obtained by optical microscopy and DSC measurements. Figure 1 demonstrates the wide range of the thermodynamically stable discotic phase.

Conoscopic observations in a polarizing microscope (Leitz Ortholux II Pol-BK) show the positive uniaxial character of the present discotic phase. No schlieren texture has been observed but a pseudo focal conic one. Thus, one can exclude the presence of an $N_{\mathrm{D}}$-phase. Consequently, the phase should be of type $\mathrm{D}_{\text {ho }}$ or $\mathrm{D}_{\mathrm{hd}}$. To distinguish between these two phases, X-ray measurements of oriented samples were carried out.

The small angle X-ray patterns of an oriented as well as an unoriented sample of the decanoyl-homologous HASI obtained by a Kiessig camera and film registration are shown in Figure 2.

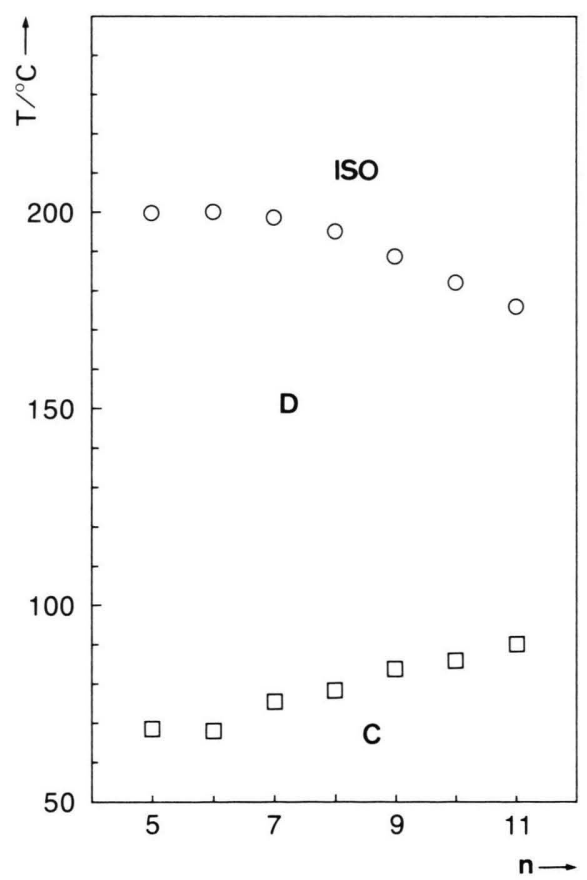

Fig. 1. Phase transition temperatures of HASI derivatives: $n=$ number of carbon atoms of the alkyl chains. (O) discotic-iso; ( $\square$ ) cryst.-discotic.
In the oriented sample a narrow ring at lower angles is to be seen, corresponding to the intercolumnar distance $d$ of regularly spaced columns. From the six spots within the inner ring it follows that the shape of the unit cell corresponds to a hexagonal lattice. According to previous results [6], a ring at larger angles represents the distance of the alkyl chains in adjacent molecules within a column. It corresponds to an average spacing of $s=0.475 \mathrm{~nm}$, which is constant in all compounds of the present homologous series. The sharpness of an intense ring at larger angles indicates a regular intercore stacking distance within the columns. Consequently, the discotic phase should be of $D_{h o}$ type.

The intercolumnar distances $d$ obtained by PSPC (Fig. 3) exhibit a linear increase with increasing alkyl chain length. The molecular diameter 1 in the

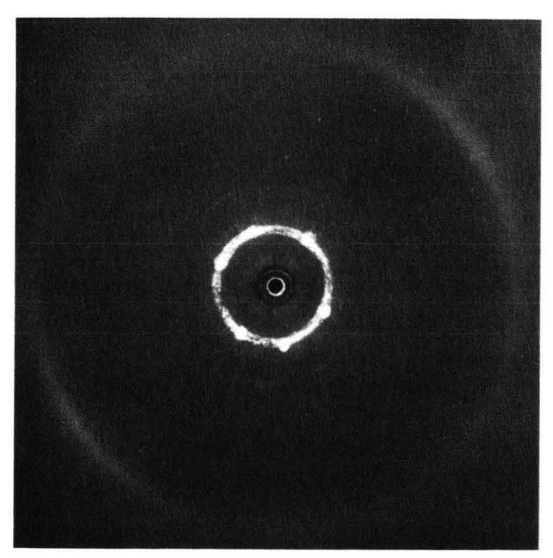

a)

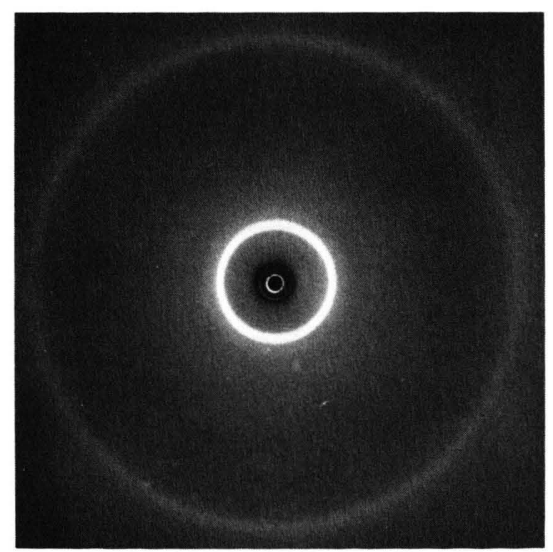

b)

Fig. 2. Small angle X-ray patterns of hexa-O-decanoylscyllo-inositol.

a) oriented sample, b) unoriented sample. 
most extended form (alkyl chains fully stretched) were calculated by means of molecular models (Dreiding) and are also plotted in Figure 3. Surprisingly, also the slope of $d(n)$ was found to be much smaller than $l(n)$. That means that $d$ increases much less with increasing alkyl chain length then $l$ does. The absolute values of $d$ are much smaller than those of $l$.

This result is important to discuss the role of the alkyl chain orientation within the adjacent columns.

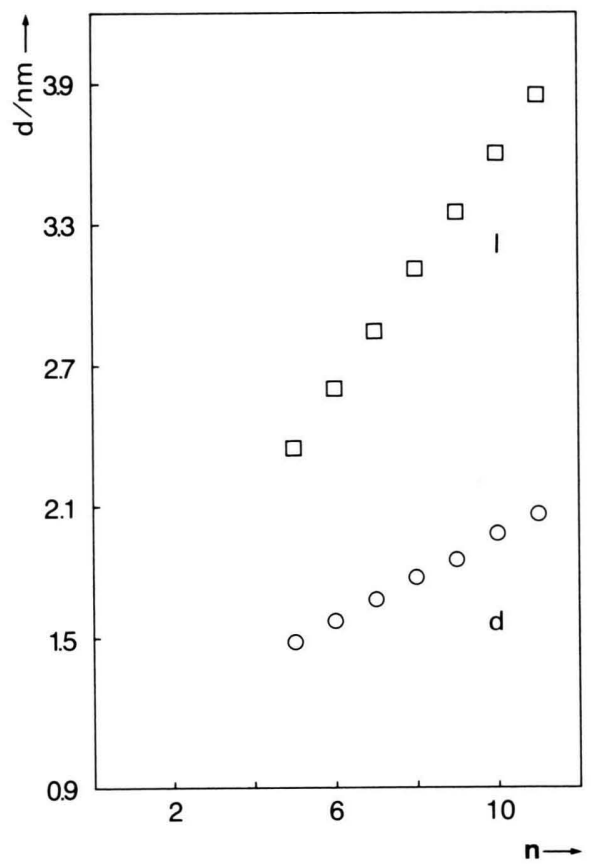

Fig. 3. Intercolumnar distance $d$ and molecular diameter $l$ vs. alkyl chain length.

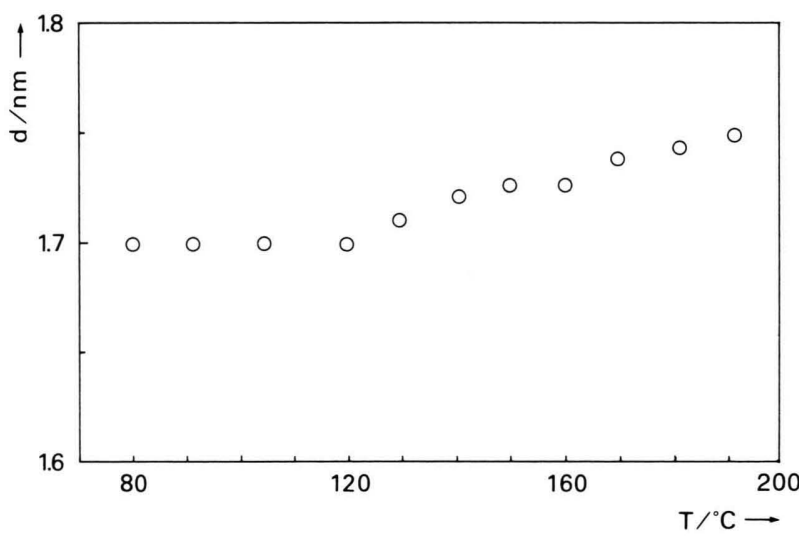

Fig. 4. Temperature dependence of $d$ in hexa-O-heptanoylscyllo-inositol.
The temperature dependence of the intercolumnar distance $d$ has been measured using PSPC. As a typical example, the results for hexa-O-heptanoylscyllo-inositol $(n=7)$ are given in Figure $4 . d$ increases with increasing temperature, but the temperature dependence of $d$ is extremely small and can be neglected at a first approximation.

\section{Volumetric Model Calculations}

In combination with the X-ray measurements, the molar volume is a suitable parameter to get information about the role of the alkyl chains in the molecular arrangement of mesophases. Unfortunately, direct measurements of the molar volume could not be performed because of the large amounts of material necessary for such measurements. Therefore, the molecular volumes of the homologous HASI molecules have been calculated by the following three different methods using standard intramolecular distances or data obtained from our X-ray measurements:

i) From Dreiding stereo models we have calculated the minimum volume $V_{\mathrm{i}}$. To obtain a value for this volume $V_{\mathrm{i}}$ of an isolated HASI molecule we used a model of hexa-O-acetyl-scyllo inositol (see Fig. 5) and first calculated the core volume $V_{\mathrm{c}}$. In a good approximation the shape of this model is a circular cone as shown in Figure 5.
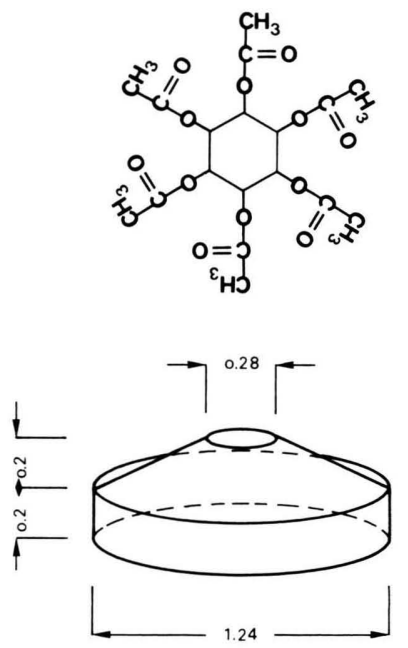

Fig. 5. Shape of hexa-O-acetyl-scyllo inositol (all distances in $\mathrm{nm})$. 

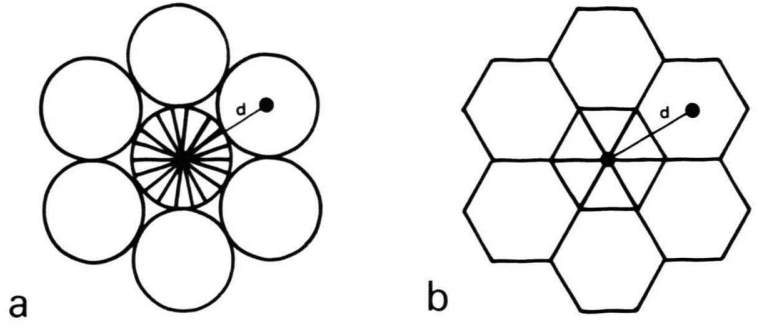

Fig. 6. Models of a hexagonal molecular packing assuming a) "free" rotation, b) strong hindered rotation.

Inserting subsequently methylene groups between the $\mathrm{CO}$ - and the $\mathrm{CH}_{3}$-group the volume $V_{\mathrm{i}}$ of the resulting HASI derivatives can be calculated by addition of the volume of the appropriate number of methylene-groups $\left(V_{\mathrm{m}}\right)$ of the six lateral alkyl chains to $V_{\mathrm{c}}$ :

$$
V_{\mathrm{i}}=V_{\mathrm{c}}+6(n-1) \cdot V_{\mathrm{m}}
$$

with

$V_{\mathrm{c}}=0.2 \pi / 4\left((0.28 / 2+1.24 / 2)^{2}+1.24^{2}\right)=0.3323 \mathrm{~nm}^{3}$

and $V_{\mathrm{m}}=0.0169 \mathrm{~nm}^{3}[7]$.

ii) On the assumption that the molecular orientation with respect to the six-fold axis is statistical and that the intercolumnar distance $d$ is twice the radius of the resulting circle (see Fig. 6) we calculated the molecular volume $V_{\mathrm{i}}$ from the X-ray diffraction data for $d$ and $s$ by means of the equation

$$
V_{\mathrm{i}}=(\pi / 4) d^{2} s .
$$

iii) Assuming a strongly hindered molecular rotation around the six-fold axis, another molecular volume $V_{2}$ can also be calculated from the X-ray data. In this case the isolated molecules describe a hexagon. The in-circle of this hexagon is identical to the circle in case ii) (see Figure 6). Therefore $d$ is twice the radius of the in-circle. The values for $V_{2}$ are obtained using the equation:

$$
V_{2}=1.5 \tan (\pi / 6) d^{2} s
$$

$d$ means the intercolumnar distance and $s=0.475 \mathrm{~nm}$ the alkyl chain intracolumnar spacing ((2) and (3)).

The results of our volumetric model calculations are given in Figure 7. Obviously, there is a linear increase of the minimum volume $V_{\mathrm{i}}$ and a quadratic one of $V_{1}$ and $V_{2}$ with increasing alkyl chain length.

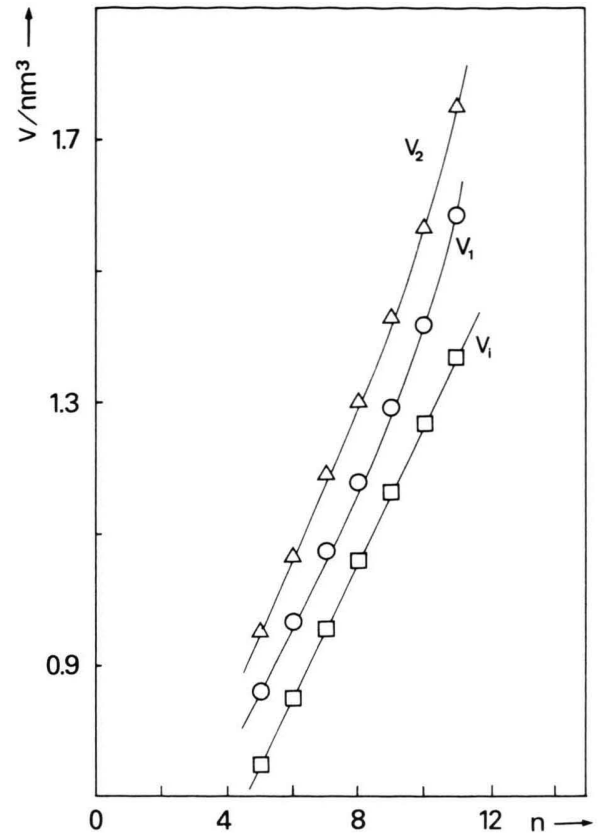

Fig. 7. Molecular volume vs. alkyl chain length.

Two experimental results must be considered in our discussion:

a) The volume increase with $n$ is similar in the three cases given above (cf. Fig. 7) with the absolute values of $V_{1}$ and $V_{2}$ deviating only by 5 to $10 \%$ from $V_{\mathrm{i}}$;

b) the slope of $d(n)$ is much smaller than that of $l(n)$, cf. Figure 3.

From these data one can derive that the alkyl chain packing becomes more dense with increasing chain length. It is obvious that the chain flexibility increases with the chain length. This has also been demonstrated by Luz et al. [8], who derived from $\mathrm{H}^{2}$-NMR data a remarkable decrease of the order parameter of the methylene groups which increasing distance from the discotic core. Taking into account this chain flexibility our data can be understood in terms of an alkyl chain interpenetration of adjacent columns. This interpenetration will be the easier the longer the chain becomes and leads to a molecular arrangement in the columnar phase with best space filling as given in Figure 8. Our model would also explain the high viscosity of the investigated discotic phase. Presently we do not know whether a more or less free rotation of the molecules is allowed or not, so we cannot distinguish between the model calculations of $V_{1}$ 


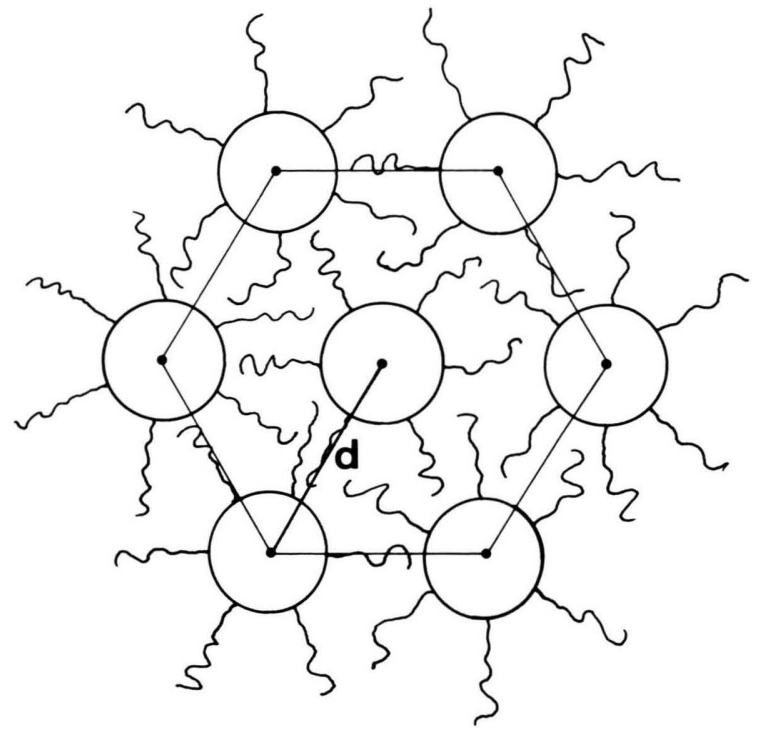

Fig. 8. Molecular arrangement of the discotic phase of scyllo inositol hexaesters.

[1] A. M. Levelut, J. Chim. Phys. 80, 149 (1983).

[2] C. Destrade, H. Gasparoux, P. Foucher, Nguyen Huu Tinh, J. Malthete, and J. Jacques, J. Chim. Phys. 80, 137 (1983).

[3] B. Kohne and K. Praefcke, Angew. Chem. 96, 70 (1984).

[4] K. B. Blodgett, J. Am. Chem. Soc. 57, 1007 (1935).

[5] A. Matsuda, J. Appl. Phys. 48, 771 (1977).

[6] P. Foucher, C. Destrade, Nguyen Huu Tinh, J. Mal- and $V_{2}$. These results support the considerations concerning the discogenity given in an earlier paper [9]: an optimal coverage of the area in the core periphery and a perfect space filling with minimum dead space in the center of the molecules are necessary for the formation of a discotic mesophase*.

\section{Acknowledgements}

This work has been supported by the Deutsche Forschungsgemeinschaft, the Ministerium für Wissenschaft und Forschung des Landes NordrheinWestfalen, the Fonds der Chemischen Industrie, and the Technische Universität Berlin.

* Note added in proof: The identification of the HASI discotic phase as a $D_{\text {ho }}$ type reported in this paper confirms earlier determinations of this type of discophase by miscibility studies of this homologous series of scylloinositol hexaesters with an authentic sample [10].

thete, and A. M. Levelut, Mol. Cryst., Liq. Cryst. 108, 219 (1984).

[7] A. Bondi, J. Phys. Chem. 68, 441 (1964).

[8] D. Goldfarb, Z. Luz, and H. Zimmermann, Israel J. Chem. 23, 341 (1983).

[9] B. Kohne and K. Praefcke, Chem. Ztg. 109, 121 (1985).

[10] B. Kohne, K. Praefcke, and J. Billard, Z. Naturforsch. 41 b, 1036 (1986). 